\title{
Mechanism of Particle Collision in the One-Dimensional Dynamics of Gas-Particle Mixtures
}

\author{
Frank E. Marble \\ Kárman Laboratory of Fluid Mechanics and Jet Propulsion \\ California Institute of Technology, Pasadena, California
}

(Received 20 February 1964)

\begin{abstract}
A theory is developed for the one-dimensional flow of a gas, containing solid particles of two different sizes, in which the effect of particle collisions is accounted for as well as the interaction between the particles and the gas. It is assumed that the particles behave as smooth elastic spheres, that they follow the Stokes drag law and exchange heat with the gas at a Nusselt number of unity. It is shown that there exists a range of parameters which provides that (i) the viscous flow fields about each particle do not interfere during collision, and (ii) the random velocities imparted by one collision are damped before either particle suffers another collision. Using the assumption of small particle slip, the one-dimensional flow problem is solved explicitly up to first order terms in the small slip. It is found, of course, that the tendency of collisions is to cause the two particle-slip speeds to have more nearly the same value than they would in the absence of interparticle collision. It appears that, although the physical assumptions restrict the magnitude of the interparticle forces, the model does provide the proper limit for very strong particle interaction and can conceivably be applied in this range also without gross error.
\end{abstract}

\section{INTRODUCTION}

$\mathrm{T}$ HE motion of clouds of small solid particles through gases is governed by the viscous forces exerted upon the particles by the relative gas motion and by the collisions between individual solid particles. Previous investigations ${ }^{1-4}$ into the dynamics of such heterogeneous systems have suppressed the effects of particle-particle collisions for either one of two reasons. If the particle number density is sufficiently small, the momentum exchange caused by collisions, and the force resulting from it, is small in comparison with the viscous resistance to particle motion. On the other hand, if the particles are of very nearly the same size, they have very nearly the same velocity relative to the gas and consequently have little tendency to collide.

Usually, neither of these two conditions is satisfied and the effects of particle-particle interactions must be accounted for in describing the motion of the mixture. In general, such a description augments the difficulties of noninteracting gas-particle flow with problems similar to those of rarified gas dynamics. There exist circumstances, however, under which the frictional damping of the particle motion simplifies the treatment of collisional processes and a

${ }^{1}$ G. F. Carrier, J. Fluid Mech. 4, 376 (1955).

2 F. E. Marble, in Proceedings of the Fifth AGARD Combustion and Propulsion Colloquium, (Pergamon Press, Ltd., London, 1963)

${ }^{3}$ W. D. Rannie, in Progress in Astronautics and Rocketry (Academic Press Inc., New York, 1962), Vol. 6.

${ }^{4}$ F. E. Marble, AIAA J. 1, 2793 (1963). general solution to the problem becomes possible. These circumstances can be seen from the following considerations.

When macroscopic particles of a single size are transported by a gas, the particles tend to assume a local slip velocity relative to the gas, the magnitude of which is proportional to the local total acceleration of the gas. A particle whose slip velocity is modified will return to its original slip velocity in a characteristic time, the velocity equilibration time. When the gas contains particles of two distinct sizes, groups of particles of each size slip at their own appropriate speed with the consequence that particles of different sizes may collide. The collision frequency determines another characteristic time, namely, the time between successive collisions suffered by a given particle. If the velocity equilibration time is short in comparison with the collision time, a particle that is disturbed from its local slip velocity by a collision will return to its local slip velocity before it encounters another particle. Consequently, the calculation of mean momentum and energy exchange between clouds of different size particles may be carried out without reference to details of previous encounters.

The proper ordering of the velocity equilibration time and collision time to permit the simplification mentioned above is not a universal occurrence; there are circumstances of considerable interest, however, where these conditions are met. It is the purpose of the present paper to examine in considerable detail 
the application of this model to a gas flow containing a particle cloud that is restricted to particles of two distinct sizes.

\section{MECHANISM OF GAS-PARTICLE FLOW WITH PARTICLE INTERACTION}

Consider a medium consisting of a gas and of clouds of two different size particles. From the multifluid viewpoint, one may recognize the important interactions between components as (1) forces between each particle cloud and the gas; (2) heat transfer between each particle cloud and the gas; (3) forces acting between the two particle clouds as a result of collisions. Heat transfer between particles as a result of collision is explicitly neglected.

It will be assumed that the individual particle motion through the gas obeys the first-order Stokes law and that the heat transfer between a particle and the gas takes place with a Nusselt number of unity. While this assumption may impair the accuracy in some circumstances, it does not modify the significant physical features of the flow.

\section{Particle-Gas Interaction}

Denote the local gas speed $u$ and the speed of an individual particle $u_{\mathrm{p}}$. The force $\phi$ exerted upon that particle by the gas is

$$
\phi=6 \pi \sigma \mu\left(u-u_{\mathrm{p}}\right)
$$

where $\sigma$ is the particle radius and $\mu$ is the gas viscosity. The total force exerted by the $n$ particles per unit volume is $n \phi$. Moreover, if $m$ is the mass of a single particle, $\rho_{\mathrm{p}} \equiv n m$ is the local mass density of the solid phase. The total force acting between the two phases per unit volume may be written

$$
F \equiv n \phi=\rho_{\mathrm{p}}(6 \pi \sigma \mu / m)\left(u-u_{\mathrm{p}}\right) .
$$

The quantity $m / 6 \pi \sigma \mu$ has the dimension of time, and from the equation of motion for a single particle

$$
m d u_{\mathrm{p}} / d t=\phi
$$

it appears that

$$
\tau_{v} \equiv m / 6 \pi \sigma \mu
$$

is the time required for the particle slip velocity $u_{\mathrm{s}} \equiv u-u_{\mathrm{p}}$ to decay to $1 / e$ of its initial value. As indicated in Ref. 2 , it is sometimes more convenient to deal with a characteristic length

$$
\lambda_{v}=m u_{0} / 6 \pi \sigma \mu=\tau_{v} u_{0}
$$

where $u_{0}$ is a velocity characteristic of the gasdynamic flow problem. The parameter $\lambda_{v}$ is denoted the velocity equilibration length and is a measure of the distance that a particle of radius $\sigma$ will be transported in a stream of velocity $u_{0}$ before its initial slip velocity decreases to $1 / e$ of its initial value.

To the same degree of accuracy the rate at which heat is transferred to a single particle at temperature $T_{\mathrm{p}}$ from the gas at temperature $T$ is

$$
q=(k / \sigma) 4 \pi \sigma^{2}\left(T-T_{\mathfrak{p}}\right)
$$

where $k$ is the thermal conductivity of the gas. The total rate of heat transfer per unit volume becomes

$$
Q \equiv n q=\rho_{\mathrm{p}} c_{p}\left(4 \pi \sigma k / m o_{p}\right)\left(T-T_{\mathrm{p}}\right)
$$

where $c_{p}$ is the specific heat at constant pressure for the gas. Analogous to the velocity equilibration time there exists a thermal equilibration time

$$
\tau_{\mathbf{T}} \equiv m c_{p} / 4 \pi \sigma k=\frac{3}{2}\left(c_{p} \mu / k\right) \tau_{v}
$$

Since the gas Prandtl number $c_{p} \mu / k$ is very nearly equal to $\frac{2}{3}$, the velocity and thermal equilibration times are nearly equal. Consequently, the times or lengths required for a particle to reach equilibrium velocity and temperature of the gas are approximately equal.

When the equilibration lengths are small, the relationship between particle and gas motion (and temperature change) may be simplified. The acceleration term in the equation of motion for a single particle in one dimension,

$$
u_{\mathrm{p}} d u_{\mathrm{p}} / d x=u_{0}\left(u-u_{\mathrm{p}}\right) / \lambda_{v}
$$

may be approximated as $u_{\mathrm{p}} d u_{\mathrm{p}} / d x \approx u d u / d x$ so that the local slip velocity $u-u_{\mathrm{p}}$ is nearly proportional to the local gas acceleration. Then if $L$ is the length over which the gas velocity changes by its characteristic velocity, the particle slip velocity is of the order

$$
\left(u-u_{\mathrm{p}}\right) / u_{0} \sim \lambda_{v} / L .
$$

Therefore, the criterion for small slip is that $\lambda_{v}$ shall be small compared with the characteristic length of the flow field.

It is instructive to observe the physical behavior of an isolated particle in an accelerated gas stream. The gas velocity is represented by the broken line in Fig. 1. A particle injected into the stream with a velocity $u_{1}$, less than the local gas velocity, experiences a drag force tending to accelerate it toward the gas velocity. This acceleration continues until the drag force decreases to that required to impart to the particle a local acceleration equal to that of the gas. On the other hand, a particle injected into the stream with a velocity $u_{2}$ greater than the local gas speed will first decelerate toward 


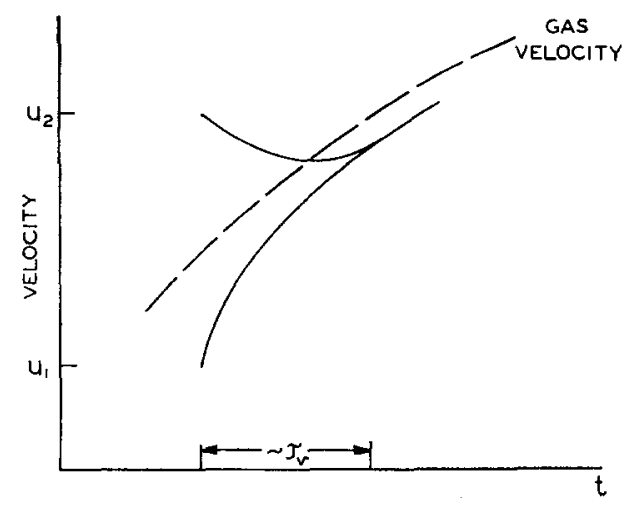

FIG. 1. Particle equilibration.

the local gas speed. Gradually the rising gas velocity will exceed the particle velocity and the particle velocity lag will increase until the same local quasiequilibrium is reached. The distance through which the particle travels before reaching the equilibrium slip condition is of the order $\lambda_{v}$ which is assumed small in comparison with interesting physical lengths of the problem. A completely parallel discussion holds for the relationship between local particle and gas temperatures.

\section{Particle-Particle Interactions}

Consider a perfect gas containing number densities $n_{1}$ and $n_{2}$ of particles having radii $\sigma_{1}$ and $\sigma_{2}$ respectively, $\sigma_{1}>\sigma_{2}$. Then these particles will have different velocity equilibration lengths $\lambda_{v}\left(\sigma_{1}\right)$ and $\lambda_{v}\left(\sigma_{2}\right)$ and consequently will have different mean slip velocities $u_{\mathrm{B}}\left(\sigma_{1}\right)$ and $u_{\mathrm{B}}\left(\sigma_{2}\right)$ at any point of the flow. If the gaseous acceleration is positive in the direction of positive $x$ axis, the slip velocities will be positive and $u_{\mathrm{s}}\left(\sigma_{1}\right)>u_{\mathrm{s}}\left(\sigma_{2}\right)$. As indicated in Fig. 2, the relative velocity of these two particle sizes will lead to collisions between particles of different sizes; in the present example it will appear that the particles of smaller radius $\sigma_{2}$ overtake particles of larger radius $\sigma_{1}$.

The frequency $N\left(\sigma_{1}\right)$ with which particles of radius $\sigma_{1}$ experience collisions may be calculated in the classical manner:

$$
N\left(\sigma_{1}\right)=n\left(\sigma_{1}\right) \pi\left(\sigma_{1}+\sigma_{2}\right)^{2}\left|u_{\mathrm{p}}\left(\sigma_{2}\right)-u_{\mathrm{p}}\left(\sigma_{1}\right)\right| .
$$

The absolute value of the velocity difference merely indicates that the number of collisions is positive regardless of the direction from which the particles approach. The time $\tau_{0}\left(\sigma_{1}\right)$ to be expected between successive encounters experienced by a particle of radius $\sigma_{1}$ is then

$$
\tau_{\mathrm{e}}\left(\sigma_{1}\right)=1 / N\left(\sigma_{1}\right),
$$

and it is of interest to compare this time with $\tau_{v}\left(\sigma_{1}\right)$, that required for velocity equilibration of the same particle. Referring to Eq. (3), this ratio of characteristic times becomes

$$
\begin{array}{r}
\frac{\tau_{v}\left(\sigma_{1}\right)}{\tau_{\mathrm{c}}\left(\sigma_{1}\right)}=\frac{1}{6}\left(\frac{\left(\sigma_{1}+\sigma_{2}\right)}{\left|u_{\mathrm{p}}\left(\sigma_{1}\right)-u_{\mathrm{p}}\left(\sigma_{2}\right)\right|}\right. \\
\nu \\
\cdot\left(\frac{\rho_{\mathrm{p}}\left(\sigma_{2}\right)}{\rho}\right)\left(\frac{\left(\sigma_{1}+\sigma_{2}\right) \sigma_{1}^{2}}{\sigma_{2}^{3}}\right)
\end{array}
$$

and the magnitude of each of the bracketed terms may be estimated. The quantity

$$
\left(\sigma_{1}+\sigma_{2}\right)\left|u_{\mathrm{p}}\left(\sigma_{1}\right)-u_{\mathrm{p}}\left(\sigma_{2}\right)\right| / \nu
$$

is a Reynolds number based upon the difference in slip velocities. Since the Reynolds number of the individual particles must be of order one for the Stokes approximation to be valid, this bracket must be at most of order one. The quantity $\rho_{\mathrm{p}}\left(\sigma_{2}\right) / \rho$, the ratio of mass density due to particles of radius $\sigma_{2}$ to the local gas density, is generally small compared with unity. The last term involves only the two particle radii and becomes large as $\sigma_{1} / \sigma_{2}$ becomes large. If it is generally agreed to restrict the product $\left[\rho_{\mathrm{p}}\left(\sigma_{2}\right) / \rho\right]\left(\sigma_{1} / \sigma_{2}\right)^{3}$ to be at most of order unity, there is no difficulty in assuring that $\tau_{v}\left(\sigma_{1}\right) / \tau_{0}\left(\sigma_{1}\right) \leq 1$.

If a given particle were subject only to viscous interaction with the gas, it would slip relative to the gas at a rate such that drag force accelerates the particle at very nearly the local gas acceleration rate. A particle, disturbed from this state by an extraneous impulse, will return to the appropriate local state of slip within a time of order $\tau_{v}$, the velocity equilibration time. Now the particle-particle interactions may be considered impulsive forces which are continually disturbing the particle from the local equilibrium slip condition which would hold in the absence of collisions. The scattering velocities that result from collisions will have components normal and tangential to the directions of

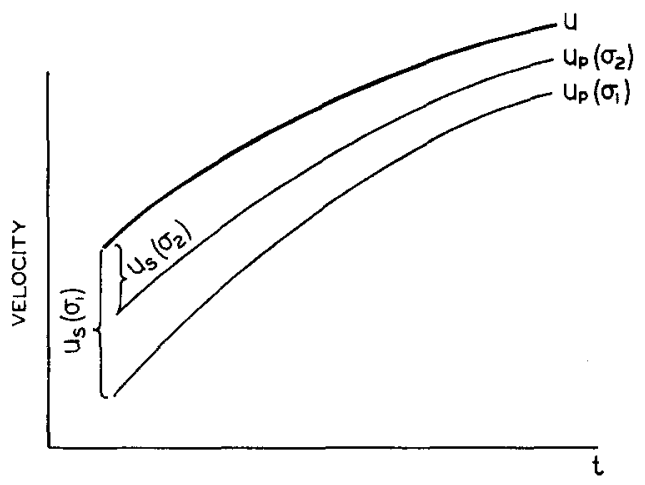

FIG. 2. Relative particle slip velocity. 
average gas and particle motions, and these are shown schematically in Figs. 3(a) and 3(b) as functions of time. The positions of successive collisions are marked along the time axes, the origin taken at the time of one collision.

Consider first the scattering velocity component normal to the average motion as indicated in Fig. 3(a). Velocity components of opposite sign are imparted to particles of radius $\sigma_{1}$ and $\sigma_{2}$ and these velocity components proceed to decay exponentially toward zero. The time constants for these decay processes are $\tau_{v}\left(\sigma_{1}\right)$ and $\tau_{v}\left(\sigma_{2}\right) ; \tau_{v}\left(\sigma_{1}\right)>\tau_{v}\left(\sigma_{2}\right)$ since $\sigma_{1}>\sigma_{2}$. If the time interval $\tau_{0}\left(\sigma_{1}\right)$ between successive collisions experienced by particle $\sigma_{1}$ is larger than or equal to the velocity equilibration time $\tau_{v}\left(\sigma_{1}\right)$, the particle may be assumed to enter into each collision with only negligible residual scattering velocity normal to the direction of average motion.

The component of scattering velocity parallel with the mean motion, Fig. $3(\mathrm{~b})$, presents a bit more complication in its understanding. By $u_{\mathbf{p}}\left(\sigma_{1}\right)$ and $u_{\mathrm{p}}\left(\sigma_{2}\right)$ we denote the velocities of particles of radius $\sigma_{1}$ and $\sigma_{2}$, each averaged over the appropriate particle class to remove fluctuations associated with collisions. The velocity of a single particle, however, may vary with time in response to the time variations of gas velocity. After a collision, the parallel components of the particle velocities are altered from their mean values as shown for example at time $t=0$ in Fig. 3(b). Subsequently the velocities of these two particles decay, not toward their average velocities, but toward their respective collisionless slip velocities, that is, the velocities these particles would experience in the same local gas flow but in the absence of collisions. These collisionless slip velocities are denoted $u_{\mathrm{p}}^{\prime}\left(\sigma_{1}\right)$ and $u_{\mathrm{p}}^{\prime}\left(\sigma_{2}\right)$; the time constants of the decay processes of the particle pair are $\tau_{v}\left(\sigma_{1}\right)$ and $\tau_{v}\left(\sigma_{2}\right)$. Consequently, the restrictions that $\tau_{v}\left(\sigma_{1}\right) / \tau_{\mathrm{c}}\left(\sigma_{1}\right) \leq 1$, $\tau_{v}\left(\sigma_{2}\right) / \tau_{\mathrm{c}}\left(\sigma_{2}\right) \leq 1$ imply that the particles of radii $\sigma_{1}$ and $\sigma_{2}$ are moving very nearly with the velocities $u_{\mathrm{p}}^{\prime}\left(\sigma_{1}\right)$ and $u_{\mathrm{p}}^{\prime}\left(\sigma_{2}\right)$ respectively before each collision.

Thus the restrictions which have been placed upon present analysis, namely,

$$
\begin{aligned}
\lambda_{r}\left(\sigma_{1}\right) / L, & \lambda_{v}\left(\sigma_{2}\right) / L \ll 1, \\
\tau_{v}\left(\sigma_{1}\right) / \tau_{\mathbf{c}}\left(\sigma_{1}\right), & \tau_{v}\left(\sigma_{2}\right) / \tau_{\mathbf{c}}\left(\sigma_{2}\right) \leq 1,
\end{aligned}
$$

ensure the following simplifications in the physical picture of the gas-particle flow process:

(i) the collisionless slip velocities are determined by the local acceleration of the equilibrium gasparticle flow field;

(ii) the random velocities resulting from one inter-

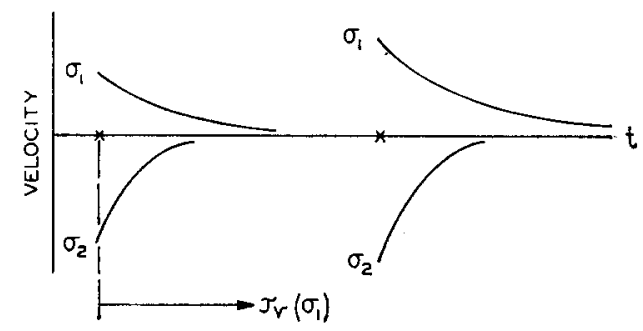

(a)

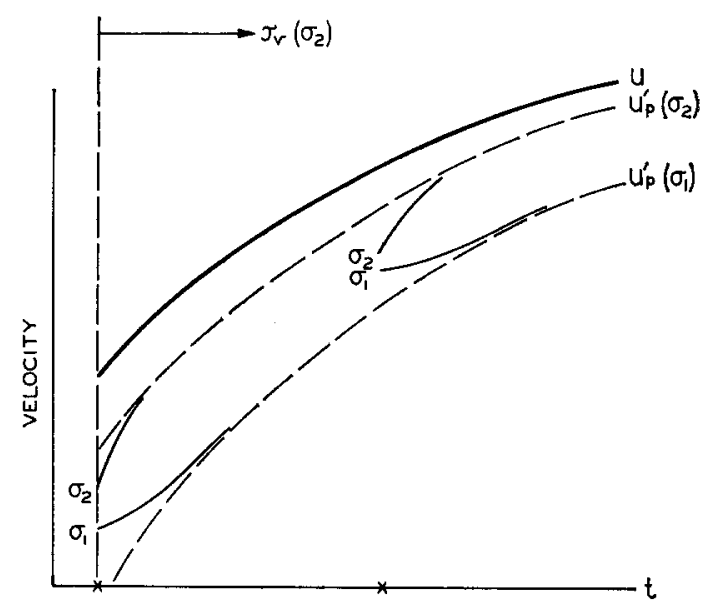

(b) FIG. 3. Decay of normal and parallel components of
scattering velocity.

particle collision have essentially decayed to zero before the next collision takes place;

(iii) all collisions take place with a relative velocity equal to the difference between the collisionless slip velocities of the two particle sizes and with a direction parallel to the local gas acceleration.

\section{Interparticle Forces}

The collisions between solid particles of different sizes result in a gross momentum exchange between the two particle classes and we may thus say that these clouds exert a force upon each other when they are considered as continuous media. It is instructive to consider a specific collisional model in detail, realizing that it may be altered or generalized rather obviously.

The first point of concern is the effect of local flow fields about the particles. As two particles approach within a few radii of each other, the force exerted by the gas upon each particle is altered by the change in local flow field caused by the other. It will specifically be assumed that this local modification to the particle force has a negligible effect upon the collision, that is, the alteration to the momentum exchange process is small. The condition for this assumption to be valid is that the time re- 
(a)

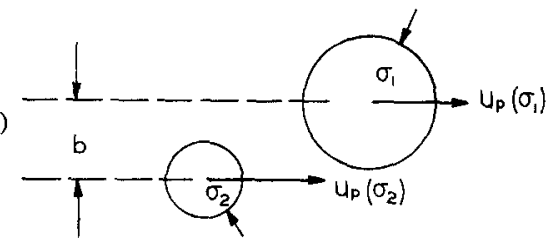

(b)

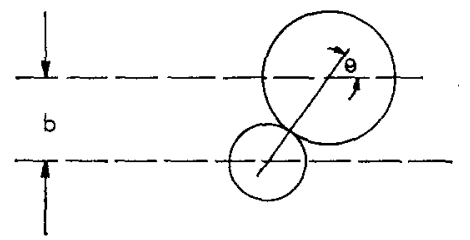

FrG. 4. Collision geometry.

quired for one particle to traverse the flow field of the other be negligible in comparison with the velocity equilibration time $\tau_{v}(\sigma)$. Now, since the time the two particles spend in their mutual flow fields is of the order $\sigma /\left|u_{\mathrm{p}}^{\prime}\left(\sigma_{1}\right)-u_{\mathrm{p}}^{\prime}\left(\sigma_{2}\right)\right|$, the conditions may be written analytically as

$$
\frac{\tau_{v}(\sigma)}{\sigma /\left|u_{\mathrm{p}}^{\prime}\left(\sigma_{1}\right)-u_{\mathrm{p}}^{\prime}\left(\sigma_{2}\right)\right|} \gg 1,
$$

where $\sigma$ and $\tau_{v}(\sigma)$ may refer to either particle. Utilizing the definition of $\tau_{v}(\sigma)$ and denoting by $\rho_{\mathrm{s}}$ the mass density of the material from which the particles are made, this may be written

$$
\frac{2}{9}\left(\frac{\rho \sigma\left|u_{\mathrm{p}}^{\prime}\left(\sigma_{1}\right)-u_{\mathrm{p}}^{\prime}\left(\sigma_{2}\right)\right|}{\mu}\right)\left(\frac{\rho_{\mathrm{s}}}{\rho}\right) \gg 1 .
$$

The first bracketed term is a Reynolds number based upon the relative velocity of the two particles; from the restriction associated with Stokes drag law, this is at most of order unity. Consequently, the inequality can be satisfied only if the density ratio $\rho_{s} / \rho$ is large, for example, by solid particles in a gaseous medium.

The second point deals with the nature of the encounter between the particle surfaces. It will be assumed that the particles are spherical, smooth, and that the collision may be considered perfectly elastic. Each of these conditions may be relaxed to a considerable extent, changing the basic problem in detail but not in principle.

Under the above assumptions, the calculation of the motion immediately following an elastic collision is a classical one. The particles move parallel with the $x$ axis with velocities $u_{\mathrm{p}}^{\prime}\left(\sigma_{1}\right)$ and $u_{\mathrm{p}}^{\prime}\left(\sigma_{2}\right)$ along lines of centers separated by a distance $b$ as shown in Fig. 4(a). The collision takes place, as indicated in Fig. 4(b), so that a line connecting the centers of the particles makes an angle $\vartheta$ with respect to the $x$ axis. The single collision parameter $b$ may then be replaced by the angle $\vartheta$. Immediately after collision the particles have velocities $u_{\mathrm{p}}^{*}\left(\sigma_{1}\right)$, $v_{\mathrm{p}}^{*}\left(\sigma_{1}\right), u_{\mathrm{p}}^{*}\left(\sigma_{2}\right), v_{\mathrm{p}}^{*}\left(\sigma_{2}\right)$ which depend only upon $\vartheta$ when the particle radii and approach velocities are known. The velocities $v_{\mathrm{v}}^{*}\left(\sigma_{1}\right)$ and $v_{\mathrm{v}}^{*}\left(\sigma_{2}\right)$ normal to the gas acceleration contribute no net momentum transfer. The momentum exchange parallel with the gas acceleration is the origin of the force acting between the two aggregates of particles. The momentum increase of a particle with radius $\sigma_{1}$, due to a single collision with collision angle $\vartheta$, is

$$
\begin{aligned}
& m\left(\sigma_{1}\right)\left[u_{\mathfrak{p}}^{*}\left(\sigma_{1}\right)-u_{\mathfrak{p}}^{\prime}\left(\sigma_{1}\right)\right] \\
& \quad=\frac{2 m\left(\sigma_{1}\right) m\left(\sigma_{2}\right)}{m\left(\sigma_{1}\right)+m\left(\sigma_{2}\right)}\left[u_{\mathfrak{p}}^{\prime}\left(\sigma_{2}\right)-u_{\mathfrak{p}}^{\prime}\left(\sigma_{1}\right)\right] \cos ^{2} \vartheta .
\end{aligned}
$$

The force per unit volume acting between the two particle clouds is then obtained by integrating this over collision angle $0 \leq \vartheta \leq \frac{1}{2} \pi$, assuming all collisions equally probable, and summing over all particles $n\left(\sigma_{1}\right)$ and $n\left(\sigma_{2}\right)$ in a unit volume. The result is

$$
\begin{aligned}
\mathfrak{F}\left(\sigma_{1} ; \sigma_{2}\right)= & \pi n\left(\sigma_{1}\right) n\left(\sigma_{2}\right)\left(\sigma_{1}+\sigma_{2}\right)^{2} \frac{m\left(\sigma_{1}\right) m\left(\sigma_{2}\right)}{m\left(\sigma_{1}\right)+m\left(\sigma_{2}\right)} \\
& \cdot\left|u_{\mathfrak{p}}\left(\sigma_{1}\right)-u_{\mathrm{p}}\left(\sigma_{2}\right)\right|\left[u_{\mathrm{p}}^{\prime}\left(\sigma_{2}\right)-u_{\mathrm{p}}^{\prime}\left(\sigma_{1}\right)\right]
\end{aligned}
$$

where $F\left(\sigma_{1} ; \sigma_{2}\right)$ is the force per unit volume exerted upon particles of radius $\sigma_{1}$ by particles of radius $\sigma_{2}$.

This interparticle force function is completely symmetrical in particle classes $\sigma_{1}$ and $\sigma_{2}$ except for the last bracketed term, the relative velocity immediately before collision. Thus $\mathfrak{F}\left(\sigma_{1} ; \sigma_{2}\right)=-\mathfrak{F}\left(\sigma_{2} ; \sigma_{1}\right)$ which states the physically obvious fact that the forces exerted upon each cloud by the other are equal but opposite. When the gas acceleration vanishes, then $u_{\mathrm{p}}\left(\sigma_{1}\right)=u_{\mathrm{p}}\left(\sigma_{2}\right)=u_{\mathrm{p}}^{\prime}\left(\sigma_{1}\right)=u_{\mathrm{p}}^{\prime}\left(\sigma_{2}\right)=u$; that is, particle velocities are equal to the gas velocity so that the collision rate and the interparticle force vanish. As a consequence, within the limitations of the model presented here, the quantities $\left[u_{\mathrm{p}}\left(\sigma_{1}\right)-u_{\mathrm{p}}\left(\sigma_{2}\right)\right]$ and $\left[u_{\mathrm{p}}^{\prime}\left(\sigma_{1}\right)-u_{\mathrm{p}}^{\prime}\left(\sigma_{2}\right)\right]$ both change sign when the gas acceleration vanishes. Hence it is irrelevant on which term the absolute value sign is indicated in Eq. (17) so long as it appears upon one or the other. Although the physical picture employed here leads naturally to the absolute value as indicated, there is an equally clear physical picture that leads to the opposite location of absolute value. The two results are, as indicated, numerically identical.

It is convenient to introduce here another characteristic length $l$ related to this interparticle force.

$$
l=\left[m\left(\sigma_{1}\right)+m\left(\sigma_{2}\right)\right] / \rho_{0} \pi\left(\sigma_{1}+\sigma_{2}\right)^{2}
$$


where $\rho_{0}$ is a reference density for the gas. It is of the order of the density ratio $\rho_{s} / \rho_{0}$ times a typical particle radius. Introducing this length, the interparticle force may be rewritten as

$$
\begin{aligned}
\mathscr{F}\left(\sigma_{1} ; \sigma_{2}\right)= & \frac{\rho_{\mathrm{p}}\left(\sigma_{1}\right) \rho_{\mathrm{p}}\left(\sigma_{2}\right)}{\rho_{0}} \frac{1}{l} \\
& \cdot\left|u_{\mathrm{p}}\left(\sigma_{1}\right)-u_{\mathrm{p}}\left(\sigma_{2}\right)\right|\left[u_{\mathrm{p}}^{\prime}\left(\sigma_{2}\right)-u_{\mathrm{p}}^{\prime}\left(\sigma_{1}\right)\right]
\end{aligned}
$$

so that it is clear that for fixed particle mass fractions, the particle radius (and hence $l$ ) must be small in order that the interparticle forces be of significant magnitude.

\section{Magnitude of Forces}

It is of particular interest to ask under what circumstances the interparticle force may be of the same order as the viscous force and hence of interest in a practical problem. This question is easily answered by noting that

$$
\begin{aligned}
& \frac{\mathscr{F}\left(\sigma_{1} ; \sigma_{2}\right)}{F\left(\sigma_{1}\right)} \\
& \quad=\frac{\tau_{v}\left(\sigma_{1}\right)}{\tau_{c}\left(\sigma_{1}\right)} \frac{m\left(\sigma_{2}\right)}{m\left(\sigma_{1}\right)+m\left(\sigma_{2}\right)}\left(\frac{u_{\mathrm{p}}^{\prime}\left(\sigma_{2}\right)-u_{\mathrm{D}}^{\prime}\left(\sigma_{1}\right)}{u-u_{\mathrm{p}}\left(\sigma_{1}\right)}\right)
\end{aligned}
$$

so that if the two particle sizes are significantly different, the ratio of collisional-to-viscous forces is of the same order as the ratio of velocity equilibration time to collisional time. For the simplified collision model employed here, it is required that $\tau_{v}\left(\sigma_{1}\right) / \tau_{0}\left(\sigma_{1}\right)$ be less than or equal to unity. When this ratio is very small, the collisional forces are negligible in comparison with the viscous forces. As the ratio is increased, for example by increasing particle radius at the same solid mass fraction, $\tau_{v}\left(\sigma_{1}\right) / \tau_{\mathrm{c}}\left(\sigma_{1}\right)$ increases directly as the particle radius. Consequently, the collisional and viscous forces become of the same order of magnitude before the assumptions underlying the model are invalidated.

From a somewhat different point of view, the ratio of collisional to viscous forces may be stated in terms of the characteristic lengths. For using Eq. (19),

$$
\frac{\mathscr{F}\left(\sigma_{1} ; \sigma_{2}\right)}{F\left(\sigma_{1}\right)}=\frac{\rho_{\mathrm{p}}\left(\sigma_{2}\right)}{\rho_{0}} \frac{\lambda_{v}\left(\sigma_{1}\right)}{l} \frac{\left|u_{\mathrm{p}}^{\prime}\left(\sigma_{2}\right)-u_{\mathrm{p}}^{\prime}\left(\sigma_{1}\right)\right|}{u_{0}},
$$

where $\left|u_{\mathrm{p}}^{\prime}\left(\sigma_{1}\right)-u_{\mathrm{p}}^{\prime}\left(\sigma_{2}\right)\right| / u_{0}$ is of the order $\lambda / L$ and $\rho_{\mathrm{p}}\left(\sigma_{2}\right) / \rho_{0}$ is of order unity. Thus, for the two forces to be of the same order, it is necessary that

$$
l / L \sim(\lambda / L)^{2}
$$

so that $l$ is much smaller than $\lambda$.

\section{FORMULATION OF THE ONE-DIMENSIONAL STEADY-FLOW PROBLEM}

The foregoing considerations provide a sufficiently concrete basis so that the general equations of continuity, motion, and first law of thermodynamics may be written down for each component of medium consisting of a gas and two particle clouds, each made up of particles having a fixed radius. It will be assumed that all the restrictions implied in discussion of the mechanism are satisfied, and it will be shown a posteriori that this is possible. It will be assumed also that there is no chemical reaction and that there is no condensation or vaporization of the particles.

The mass flow of each phase and each particle size is conserved so that the three appropriate equations of continuity are

$$
\begin{gathered}
\rho u A=\dot{m} . \\
\rho_{p}\left(\sigma_{1}\right) u_{\mathrm{p}}\left(\sigma_{1}\right) A=\kappa\left(\sigma_{1}\right) \dot{m}, \\
\rho_{p}\left(\sigma_{2}\right) u_{p}\left(\sigma_{2}\right) A=\kappa\left(\sigma_{2}\right) \dot{m} .
\end{gathered}
$$

Here $\dot{m}$ represents the mass flow of gas through the cross-sectional area $A ; \kappa\left(\sigma_{1}\right)$ and $\kappa\left(\sigma_{2}\right)$ ratios of mass flow of particles, having radii $\sigma_{1}$ and $\sigma_{2}$ respectively, to the mass flow of the gas. The quantities $\kappa\left(\sigma_{1}\right)$ and $\kappa\left(\sigma_{2}\right)$ are constants of the motion and are considered prescribed.

The equation of motion for the gas is

$$
\rho u d u / d x+d p / d x=-F\left(\sigma_{1}\right)-F\left(\sigma_{2}\right)
$$

where $F\left(\sigma_{1}\right)$ and $F\left(\sigma_{2}\right)$, the forces per unit volume exerted by the gas upon particles of radius $\sigma_{1}$ and $\sigma_{2}$ respectively, are

$$
\begin{aligned}
& F\left(\sigma_{1}\right)=\rho_{\mathrm{p}}\left(\sigma_{1}\right)\left[u-u_{\mathrm{p}}\left(\sigma_{1}\right)\right] a / \lambda_{v}\left(\sigma_{1}\right), \\
& F\left(\sigma_{2}\right)=\rho_{\mathrm{p}}\left(\sigma_{2}\right)\left[u-u_{\mathrm{p}}\left(\sigma_{2}\right)\right] a / \lambda_{v}\left(\sigma_{2}\right) .
\end{aligned}
$$

The velocity equilibration lengths have been based upon $a$, the local velocity of sound, for if we assume the viscosity coefficient of the gas to vary as $\mu \sim T^{\frac{1}{2}}$, then

$$
\lambda_{v}\left(\sigma_{i}\right)=m\left(\sigma_{i}\right) a / 6 \pi \sigma_{i} \mu
$$

are constant. The equations of motion for the two particle clouds are

$\rho_{\mathrm{p}}\left(\sigma_{1}\right) u_{\mathrm{p}}\left(\sigma_{1}\right)(d / d x)\left[u_{\mathrm{p}}\left(\sigma_{1}\right)\right]=F\left(\sigma_{1}\right)+\mathfrak{F}\left(\sigma_{1} ; \sigma_{2}\right)$,

$\rho_{\mathrm{p}}\left(\sigma_{2}\right) u_{\mathrm{p}}\left(\sigma_{2}\right)(d / d x)\left[u_{\mathrm{p}}\left(\sigma_{2}\right)\right]=F\left(\sigma_{2}\right)-\mathscr{F}\left(\sigma_{1} ; \sigma_{2}\right)$,

where the interparticle force $\Im\left(\sigma_{1} ; \sigma_{2}\right)$ is defined by Eq. (19). It is to be noted that the equations of motion do not account for the details of gas motion about individual particles. As described at some detail in Ref. 2, this implies that the momentum 
defect introduced into the gas by particle motion is immediately diffused uniformly throughout the gas. The same phenomenon applies to the energy dissipated into the gas by particle motion through it. In microscopic detail, the energy first appears in the particle wake as a velocity disturbance which is, in turn, dissipated to thermal energy. In our approximation, this energy is dissipated immediately and uniformly throughout a local volume large with respect to the particle size.

The interparticle forces involve not only the mean particle velocities $u_{\mathrm{p}}\left(\sigma_{i}\right)$ but also the collisionless particle velocities $u_{\mathrm{p}}^{\prime}\left(\sigma_{i}\right)$. These latter velocities have been defined as the velocities that individual particles would have locally in the same gas flow in the absence of collisions. Clearly, then, they satisfy the differential equations

$$
\begin{aligned}
& u_{\mathfrak{p}}^{\prime}\left(\sigma_{1}\right)(d / d x)\left[u_{\mathfrak{p}}^{\prime}\left(\sigma_{1}\right)\right]=\left[u-u_{\mathrm{p}}^{\prime}\left(\sigma_{1}\right)\right] a / \lambda_{v}\left(\sigma_{1}\right), \\
& u_{\mathrm{p}}^{\prime}\left(\sigma_{2}\right)(d / d x)\left[u_{\mathrm{p}}^{\prime}\left(\sigma_{2}\right)\right]=\left[u-u_{\mathrm{p}}^{\prime}\left(\sigma_{2}\right)\right] a / \lambda_{\mathrm{p}}\left(\sigma_{2}\right),
\end{aligned}
$$

which describe the motion of individual particles in a one-dimensional gas stream $u(x)$.

Now the individual particles change their thermal state only through heat transfer with the gas; it is specifically assumed that there is no direct exchange of heat between particles during collision. Then if $c$ is the constant specific heat of the material composing the particles, the first law of thermodynamics applied to each particle cloud gives

$$
\begin{aligned}
& \rho_{\mathrm{p}}\left(\sigma_{1}\right) u_{\mathrm{p}}\left(\sigma_{1}\right)(d / d x)\left[0 T_{\mathrm{p}}\left(\sigma_{1}\right)\right]=Q\left(\sigma_{1}\right), \\
& \rho_{\mathrm{p}}\left(\sigma_{2}\right) u_{\mathrm{p}}\left(\sigma_{2}\right)(d / d x)\left[c T_{\mathrm{p}}\left(\sigma_{2}\right)\right]=Q\left(\sigma_{2}\right),
\end{aligned}
$$

where $Q\left(\sigma_{1}\right)$ and $Q\left(\sigma_{2}\right)$ are the rates of heat transfer per unit volume from the gas to particles of radius $\sigma_{1}$ and $\sigma_{2}$ respectively. These are defined as

$$
\begin{aligned}
& Q\left(\sigma_{1}\right)=\rho_{\mathrm{p}}\left(\sigma_{1}\right) a c_{p}\left[T-T_{\mathrm{p}}\left(\sigma_{1}\right)\right] / \lambda_{\mathrm{T}}\left(\sigma_{1}\right), \\
& Q\left(\sigma_{2}\right)=\rho_{\mathrm{p}}\left(\sigma_{2}\right) a c_{p}\left[T-T_{\mathrm{p}}\left(\sigma_{2}\right)\right] / \lambda_{\mathrm{T}}\left(\sigma_{2}\right)
\end{aligned}
$$

where, as in the case of the velocity equilibration length, the local sound speed $a$ is employed in defining $\lambda_{T}\left(\sigma_{1}\right)$ and $\lambda_{T}\left(\sigma_{2}\right)$. In particular

$$
\lambda_{\mathbf{T}}\left(\sigma_{1}\right)=m\left(\sigma_{i}\right) c_{p} a / 4 \pi \sigma_{i} k
$$

where the ratio $a / k$ is assumed independent of temperature.

The energy equation for the entire system, the gas and particles together, is easily written down in the form

$$
\begin{aligned}
& \rho u(d / d x)\left(c_{p} T+\frac{1}{2} u^{2}\right) \\
& \quad+\rho_{\mathrm{p}}\left(\sigma_{1}\right) u_{\mathrm{p}}\left(\sigma_{1}\right)(d / d x)\left[c T_{\mathrm{p}}\left(\sigma_{1}\right)+\frac{1}{2} u_{\mathrm{p}}^{2}\left(\sigma_{1}\right)\right] \\
& \quad+\rho_{\mathrm{p}}\left(\sigma_{2}\right) u_{\mathrm{p}}\left(\sigma_{2}\right)(d / d x)\left[c T_{\mathrm{p}}\left(\sigma_{2}\right)+\frac{1}{2} u_{\mathrm{p}}^{2}\left(\sigma_{2}\right)\right]=0 .
\end{aligned}
$$

From this the appropriate expression of the first law of thermodynamies for the gas alone may be obtained by utilizing the other relations we have written down. For example, multiplying Eq. (29) by $u_{\mathrm{p}}\left(\sigma_{1}\right)$ gives

$$
\begin{aligned}
& \rho_{\mathrm{p}}\left(\sigma_{1}\right) u_{\mathfrak{p}}\left(\sigma_{1}\right)(d / d x)\left[\frac{1}{2} u_{\mathrm{p}}^{2}\left(\sigma_{1}\right)\right] \\
& =u_{\mathfrak{p}}\left(\sigma_{1}\right) F\left(\sigma_{1}\right)+u_{\mathrm{p}}\left(\sigma_{1}\right) \mathscr{F}\left(\sigma_{1} ; \sigma_{2}\right),
\end{aligned}
$$

which may be utilized to remove one of the terms in Eq. (38). Equations (30), (33), and (34) may be employed in a similar manner to give

$$
\begin{aligned}
& \rho u(d / d x)\left(c_{p} T\right)-u d p / d x=-Q\left(\sigma_{1}\right)-Q\left(\sigma_{2}\right) \\
& \quad+\left[u-u_{\mathrm{p}}\left(\sigma_{1}\right)\right] F_{\mathrm{p}}\left(\sigma_{1}\right)+\left[u-u_{\mathrm{p}}\left(\sigma_{2}\right)\right] F_{\mathrm{p}}\left(\sigma_{2}\right) \\
& \quad+\left[u_{\mathrm{p}}\left(\sigma_{2}\right)-u_{\mathrm{p}}\left(\sigma_{1}\right)\right] \mathfrak{F}\left(\sigma_{1} ; \sigma_{2}\right)
\end{aligned}
$$

and the terms on the right-hand side are of particular interest. The terms $Q\left(\sigma_{1}\right)+Q\left(\sigma_{2}\right)$ have the obvious significance of the rate at which heat is transferred from the gaseous phase. The term $\left[u-u_{\mathfrak{p}}\left(\sigma_{1}\right)\right] F\left(\sigma_{1}\right)$ represents the dissipation to the gas caused by relative motion of particles having radius $\sigma_{1}$. Note in particular that, because of the definition of $F\left(\sigma_{1}\right)$, this term is always positive regardless of whether the particles move more or less rapidly than the gas. This term expresses the conversion of kinetic energy, appearing in the wake of the particles, to heat and, as mentioned earlier, it is assumed that this conversion takes place immediately and not at some distance downstream of the particle. The term $\left[u-u_{\mathrm{p}}\left(\sigma_{2}\right)\right] F\left(\sigma_{2}\right)$ has precisely the corresponding significance for particles of radius $\sigma_{2}$. Finally, the term $\left[u_{\mathrm{p}}\left(\sigma_{2}\right)-u_{\mathrm{p}}\left(\sigma_{1}\right)\right] \mathscr{F}\left(\sigma_{1} ; \sigma_{2}\right)$, which is also a positive definite quantity, represents a dissipation associated with the collision. In detail, this quantity represents the energy, dissipated in heat, involved in damping the random motions of the individual particles which they acquire by collision. It is because each pair of particles enters into a collision from certain well-defined states that this dissipation term arises to account for the energy converted to heat during their return to their uniform states.

\section{ONE-DIMENSIONAL FLOW WITH SMALL PARTICLE SLIP}

It is appropriate to consider the solution of the problem posed in the previous section under the special circumstances of small particle slip, that is, when $\lambda / L \ll 1$. This is so not only because such problems are of considerable physical interest and analytically relatively tractable, but because the mechanism that has been assumed is strictly con- 
sistent only when the slip velocities of all particle classes may be considered small. The method which has been employed extensively in treating heterogeneous flows with a single particle size, expanding in ascending power series of $\lambda / L$, will be extended to the present problem including collisions. To do this in a problem that includes two separate small lengths, $\lambda$ and $l$, requires that there be a relationship between the orders of magnitude of $\lambda$ and $l$. It will appear that this arises naturally when consideration is restricted to the circumstances under which $F\left(\sigma_{1}\right), F\left(\sigma_{2}\right)$, and $\mathscr{F}\left(\sigma_{1} ; \sigma_{2}\right)$ can be of the same order of magnitude.

Because the assumption of small slip velocity will be introduced, it is convenient to utilize some new dependent variables that emphasize the fact that the particle velocities are very nearly the gas velocities, the particle temperatures are very nearly the gas temperatures, and the densities of the two particle sizes are very nearly those that would obtain if the particles remained always associated with the same mass of gas. These variables are then

$$
\begin{gathered}
u_{\mathrm{s}}\left(\sigma_{1}\right)=u-u_{\mathrm{p}}\left(\sigma_{1}\right), \quad u_{\mathrm{s}}\left(\sigma_{2}\right)=u-u_{\mathrm{p}}\left(\sigma_{2}\right), \\
u_{\mathrm{s}}^{\prime}\left(\sigma_{1}\right)=u-u_{\mathrm{p}}^{\prime}\left(\sigma_{1}\right), \quad u_{\mathrm{s}}^{\prime}\left(\sigma_{2}\right)=u-u_{\mathrm{p}}^{\prime}\left(\sigma_{2}\right) \\
T_{\mathrm{s}}\left(\sigma_{1}\right)=T-T_{\mathrm{p}}\left(\sigma_{1}\right), T_{\mathrm{s}}\left(\sigma_{2}\right)=T-T_{\mathrm{p}}\left(\sigma_{2}\right), \\
\rho_{\mathrm{s}}\left(\sigma_{1}\right)=\kappa\left(\sigma_{1}\right) \rho-\rho_{\mathrm{p}}\left(\sigma_{1}\right), \\
\rho_{\mathrm{s}}\left(\sigma_{2}\right)=\kappa\left(\sigma_{2}\right) \rho-\rho_{\mathrm{p}}\left(\sigma_{2}\right) .
\end{gathered}
$$

Now it is possible, through introducing these variables, to establish two groups of equations. One of these pertains to the heterogeneous mixture as a whole and in them the internal momentum and energy exchange processes do not appear explicitly. The continuity equation, momentum equation, and first law of thermodynamics thus obtained are respectively

$$
\begin{aligned}
& {\left[1+\kappa\left(\sigma_{1}\right)+\kappa\left(\sigma_{2}\right)\right] \rho u A=\left[1+\kappa\left(\sigma_{1}\right)+\kappa\left(\sigma_{2}\right)\right] \dot{m}(42)} \\
& {\left[1+\kappa\left(\sigma_{1}\right)+\kappa\left(\sigma_{2}\right)\right] \rho u \frac{d u}{d x}+\frac{d p}{d x}} \\
& \quad=\kappa\left(\sigma_{1}\right) \rho u \frac{d}{d x} u_{\mathrm{s}}\left(\sigma_{1}\right)+\kappa\left(\sigma_{2}\right) \rho u \frac{d}{d x} u_{\mathrm{s}}\left(\sigma_{2}\right) \\
& \frac{c_{p}+c \kappa\left(\sigma_{1}\right)+c \kappa\left(\sigma_{2}\right)}{1+\kappa\left(\sigma_{1}\right)+\kappa\left(\sigma_{2}\right)} T+\frac{1}{2} u^{2}-\mathrm{const} \\
& =\frac{\kappa\left(\sigma_{1}\right)}{1+\kappa\left(\sigma_{1}\right)+\kappa\left(\sigma_{2}\right)}\left[c T_{\mathrm{s}}\left(\sigma_{1}\right)+u u_{\mathrm{s}}\left(\sigma_{1}\right)-\frac{1}{2} u_{\mathrm{s}}^{2}\left(\sigma_{1}\right)\right] \\
& \quad+\frac{\kappa\left(\sigma_{2}\right)}{1+\kappa\left(\sigma_{1}\right)+\kappa\left(\sigma_{2}\right)}\left[c T_{\mathrm{s}}\left(\sigma_{2}\right)+u u_{\mathrm{s}}\left(\sigma_{2}\right)-\frac{1}{2} u_{\mathrm{s}}^{2}\left(\sigma_{2}\right)\right]
\end{aligned}
$$

where the last equation has been integrated, the constant being the integration constant determined by knowledge of the gas and particle state at some point of the flow. If these are coupled with the equation of state written in the form

$p=\left[1+\kappa\left(\sigma_{1}\right)+\kappa\left(\sigma_{2}\right)\right] \rho \frac{R}{1+\kappa\left(\sigma_{1}\right)+\kappa\left(\sigma_{2}\right)} T$

it is easily seen that in the case of completely equilibrium flow, that is, when all (small) quantities with subscript $s$ are identically zero, the gas behaves like an ordinary gas having a modified density

$$
\bar{\rho}=\left[1+\kappa\left(\sigma_{1}\right)+\kappa\left(\sigma_{2}\right)\right] \rho
$$

and modified gas properties

$$
\begin{gathered}
\bar{c}_{p}=\frac{c_{p}+c_{\kappa}\left(\sigma_{1}\right)+c \kappa\left(\sigma_{2}\right)}{1+\kappa\left(\sigma_{1}\right)+\kappa\left(\sigma_{2}\right)}, \\
\bar{c}_{v}=\frac{c_{v}+c_{\kappa}\left(\sigma_{1}\right)+c \kappa\left(\sigma_{2}\right)}{1+\kappa\left(\sigma_{1}\right)+\kappa\left(\sigma_{2}\right)} \\
\bar{R}=\bar{c}_{p}-\bar{c}_{v}=\frac{R}{1+\kappa\left(\sigma_{1}\right)+\kappa\left(\sigma_{2}\right)}, \\
\bar{\gamma}=\frac{\bar{c}_{p}}{\bar{c}_{p}}=\frac{c_{p}+c_{\kappa}\left(\sigma_{1}\right)+c \kappa\left(\sigma_{2}\right)}{c_{v}+c \kappa\left(\sigma_{1}\right)+c \kappa\left(\sigma_{2}\right)} .
\end{gathered}
$$

In other words, under conditions of complete equilibrium, the particles contribute to the effective gas density, the effective thermal capacity, but not to the pressure. In fact, a glance at Eqs. (42) through (45) shows that under such conditions of equilibrium, this gas with the modified properties flows isentropically. When the slip quantities are small, but not identically zero, that is, when the process is nearly in equilibrium, the flow will be correspondingly close to an isentropic one. To emphasize this point in a manner that will prove convenient in the analysis, Eqs. (42) through (45) may be combined in a relatively straightforward manner to give a relation which is the counterpart of the conventional isentropic relation of classical thermodynamics

$$
\begin{aligned}
& \frac{d}{d x} \log \left(\frac{T}{p^{(\tilde{\gamma}-1 / / \gamma}}\right)=\frac{1}{\bar{c}_{p} T} \\
& \quad\left\{\alpha\left(\sigma_{1}\right)\left[c \frac{d T_{\mathrm{s}}\left(\sigma_{1}\right)}{d x}+u_{\mathrm{s}}\left(\sigma_{\mathrm{l}}\right) \frac{d u}{d x}-u_{\mathrm{s}}\left(\sigma_{1}\right) \frac{d u_{\mathrm{s}}\left(\sigma_{1}\right)}{d x}\right]\right. \\
& \left.+\alpha\left(\sigma_{2}\right)\left[e \frac{d T_{\mathrm{s}}\left(\sigma_{2}\right)}{d x}+u_{\mathrm{s}}\left(\sigma_{2}\right) \frac{d u}{d x}-u_{\mathrm{s}}\left(\sigma_{2}\right) \frac{d u_{\mathrm{s}}\left(\sigma_{2}\right)}{d x}\right]\right\}
\end{aligned}
$$

This relationship may be integrated along the $x$ direction from some reference point, subscript 0 , 
to an arbitrary value of $x$ to give

$$
\begin{gathered}
\frac{T}{T_{0}}\left(\frac{p_{0}}{p}\right)^{(\dot{\gamma}-1) / \dot{\gamma}}=\exp \left\{\alpha ( \sigma _ { 1 } ) \int ^ { x } \frac { 1 } { \overline { c _ { p } T } } \left[c \frac{d T_{\mathrm{s}}\left(\sigma_{1}\right)}{d x}\right.\right. \\
\left.+u_{\mathrm{s}}\left(\sigma_{1}\right) \frac{d u}{d x}-u_{\mathrm{s}}\left(\sigma_{1}\right) \frac{d u_{s}\left(\sigma_{1}\right)}{d x}\right] d x \\
+\alpha\left(\sigma_{2}\right) \int^{x} \frac{1}{\bar{c}_{p} T}\left[c \frac{d T_{\mathrm{s}}\left(\sigma_{2}\right)}{d x}+u_{\mathrm{s}}\left(\sigma_{2}\right) \frac{d u}{d x}\right. \\
\left.\left.\quad-u_{\mathrm{s}}\left(\sigma_{2}\right) \frac{d u_{\mathrm{s}}\left(\sigma_{2}\right)}{d x}\right] d x\right\},
\end{gathered}
$$

where $\alpha\left(\sigma_{i}\right)$ is the mass fraction of particles having radius $\sigma_{i}$

$$
\alpha\left(\sigma_{i}\right) \equiv \kappa\left(\sigma_{i}\right) /\left[1+\kappa\left(\sigma_{1}\right)+\kappa\left(\sigma_{2}\right)\right] .
$$

When the flow is completely in equilibrium, the slip quantities vanish identically, the right-hand side of Eq. (49) becomes unity, leaving the isentropic relation for the modified gas.

It remains to define the slip quantities which measure the departure of the particle cloud properties from those they would have were the system in equilibrium. From the equations of continuity, Eqs. (24) and (25),

$$
\begin{aligned}
& u \rho_{\mathrm{s}}\left(\sigma_{1}\right)+\kappa\left(\sigma_{1}\right) \rho u_{\mathrm{s}}\left(\sigma_{1}\right)=\rho_{\mathrm{s}}\left(\sigma_{1}\right) u_{\mathrm{s}}\left(\sigma_{1}\right), \\
& u \rho_{\mathrm{s}}\left(\sigma_{2}\right)+\kappa\left(\sigma_{2}\right) \rho u_{\mathrm{s}}\left(\sigma_{2}\right)=\rho_{\mathrm{s}}\left(\sigma_{2}\right) u_{\mathrm{B}}\left(\sigma_{2}\right) .
\end{aligned}
$$

Similarly, from the equations of motion for the two particle clouds, Eqs. (29) and (30), two appropriate relations may be evolved simply by rewriting them in terms of the slip quantities

$$
\begin{aligned}
& u \frac{d u}{d x}-\left(\frac{\rho_{\mathrm{p}}\left(\sigma_{1}\right)}{\kappa\left(\sigma_{1}\right) \rho}\right) a \frac{u_{\mathrm{s}}\left(\sigma_{1}\right)}{\lambda_{v}\left(\sigma_{1}\right)}-\left(\frac{\rho_{\mathrm{p}}\left(\sigma_{1}\right)}{\kappa\left(\sigma_{1}\right) \rho}\right) \frac{\rho_{\mathrm{p}}\left(\sigma_{2}\right)}{\rho_{0}} \frac{1}{l} \\
& \cdot\left|u_{\mathrm{s}}\left(\sigma_{1}\right)-u_{\mathrm{s}}\left(\sigma_{2}\right)\right|\left[u_{\mathrm{s}}^{\prime}\left(\sigma_{1}\right)-u_{\mathrm{s}}^{\prime}\left(\sigma_{2}\right)\right]=u \frac{d u_{\mathrm{s}}\left(\sigma_{1}\right)}{d x}, \\
& u \frac{d u}{d x}-\left(\frac{\rho_{\mathrm{p}}\left(\sigma_{2}\right)}{\kappa\left(\sigma_{2}\right) \rho}\right) a \frac{u_{\mathrm{s}}\left(\sigma_{2}\right)}{\lambda_{v}\left(\sigma_{2}\right)}+\left(\frac{\rho_{\mathrm{p}}\left(\sigma_{2}\right)}{\kappa\left(\sigma_{2}\right) \rho}\right) \frac{\rho_{\mathrm{p}}\left(\sigma_{1}\right)}{\rho_{0}} \frac{1}{l} \\
& \cdot\left|u_{\mathrm{s}}\left(\sigma_{1}\right)-u_{\mathrm{s}}\left(\sigma_{2}\right)\right|\left[u_{\mathrm{s}}^{\prime}\left(\sigma_{1}\right)-u_{\mathrm{s}}^{\prime}\left(\sigma_{2}\right)\right]=u \frac{d u_{\mathrm{s}}\left(\sigma_{2}\right)}{d x} .
\end{aligned}
$$

A corresponding set of equations results from Eqs. (31) and (32) involving the collisionless slip velocities

$$
\begin{aligned}
u \frac{d u}{d x}- & a \frac{u_{s}^{\prime}\left(\sigma_{1}\right)}{\lambda_{v}\left(\sigma_{1}\right)} \\
& =u \frac{d u_{s}^{\prime}\left(\sigma_{2}\right)}{d x}+u_{\mathrm{s}}^{\prime}\left(\sigma_{1}\right) \frac{d}{d x}\left[u-u_{\mathrm{s}}^{\prime}\left(\sigma_{1}\right)\right], \\
u \frac{d u}{d x}- & a \frac{u_{s}^{\prime}\left(\sigma_{2}\right)}{\lambda_{v}\left(\sigma_{2}\right)} \\
& =u \frac{d u_{s}^{\prime}\left(\sigma_{2}\right)}{d x}+u_{\mathrm{s}}^{\prime}\left(\sigma_{2}\right) \frac{d}{d x}\left[u-u_{\mathrm{s}}^{\prime}\left(\sigma_{2}\right)\right] .
\end{aligned}
$$

Finally, the equations expressing the first law of thermodynamics [(33) and (34)] for each particle cloud may be rewritten in the form

$$
\begin{aligned}
& u \frac{d T}{d x}-\frac{\rho_{\mathrm{p}}\left(\sigma_{1}\right)}{\kappa\left(\sigma_{1}\right) \rho} \frac{c_{p}}{c} a \frac{T_{\mathrm{s}}\left(\sigma_{1}\right)}{\lambda_{\mathrm{T}}\left(\sigma_{1}\right)}=u \frac{d T_{\mathrm{s}}\left(\sigma_{1}\right)}{d x}, \\
& u \frac{d T}{d x}-\frac{\rho_{\mathrm{p}}\left(\sigma_{2}\right)}{{ }_{\kappa}\left(\sigma_{2}\right) \rho} \frac{c_{p}}{c} a \frac{T_{\mathrm{s}}\left(\sigma_{2}\right)}{\lambda_{\mathrm{T}}\left(\sigma_{2}\right)}=u \frac{d T_{\mathrm{s}}\left(\sigma_{2}\right)}{d x} .
\end{aligned}
$$

The problem is now described by Eqs. (42), (44), (45), (49), and (51) through (58), and it is appropriate to analyze the orders of magnitude of various terms in these equations. The magnitude of any "slip term," that is, one with a subscript s, has been somewhat loosely considered small. With more precision, the ratio of any slip quantity to the reference value of that quantity is of the order of the ratio the relevant equilibration length to the characteristic physical length of the problem. Denote by $\lambda$ and $\sigma$ a typical equilibration length and particle size so that $\lambda$ is of the same order as $\lambda_{v}\left(\sigma_{1}\right) \cdots \lambda_{T}\left(\sigma_{2}\right)$ and so that $\sigma$ is of the same order as $\sigma_{1}$ and $\sigma_{2}$. As a matter of convenience, relate the two as

$$
\lambda \equiv m(\sigma) \bar{a} / 6 \pi \sigma \mu
$$

and $\bar{a}$ is the sonic velocity corresponding to equilibrium flow, $\bar{a}=(\bar{\gamma} \bar{R} T)^{\frac{1}{2}}$.

Now it is clear that all terms of Eq. (42) are zeroth order in $\lambda / L$, as are those of Eq. (45). Correspondingly, the terms of the left-hand side of Eq. (44) are of zeroth order in $\lambda / L$ while those on the right-hand side are of first or higher order. Again, the left-hand side of Eq. (49) is of zeroth order and the exponent of the right-hand side is of order $\lambda / L$. Proceeding to Eqs. (51) and (52), the particle continuity equations, the left-hand side of each is of the order $\lambda / L$ while the right-hand side is of order $(\lambda / L)^{2}$. The remaining six equations require somewhat more care in their analysis. Referring to Eq. (53), although the slip velocity $u_{\mathrm{s}}\left(\sigma_{1}\right)$ is of the magnitude $u_{0} \lambda / L$, the quotient

$$
u_{\mathrm{s}}\left(\sigma_{1}\right) / \lambda_{v}\left(\sigma_{1}\right) \sim u_{0} / L
$$

and consequently the first and second terms on the left-hand side of (53) are of zeroth order in $\lambda / L$. The third group on the left-hand side has a significant factor that may be written

$$
\frac{\lambda^{2}}{l}\left|\frac{u_{\mathrm{s}}\left(\sigma_{1}\right)-u_{\mathrm{s}}\left(\sigma_{2}\right)}{\lambda}\right|\left(\frac{u_{\mathrm{s}}^{\prime}\left(\sigma_{1}\right)-u_{\mathrm{s}}^{\prime}\left(\sigma_{2}\right)}{\lambda}\right),
$$

where the last two factors of Eq. (61) are of zeroth order in $\lambda / L$. Now if $\lambda^{2} / L l$ is of order unity, or 
stated otherwise, if

$$
l / L \sim(\lambda / L)^{2}
$$

then the third term of Eq. (53), representing the interparticle force contribution, is also of zeroth order in $\lambda / L$ and hence of the same magnitude as the other term on the left-hand of Eq. (53). But as was discussed earlier and explicitly stated in Eq. (22), interparticle forces can attain the same magnitude as the viscous forces. Since this has been assumed already, it may be concluded that Eq. (62) is satisfied. The result is that all terms on the lefthand side of Eq. (53) are of zeroth order in $\lambda / L$, while all of those on the right-hand side are of order $\lambda / L$ or higher. The same may be said, through identical or similar arguments, of Eqs. (54) through (58).

Under the restrictions of Eq. (62), the problem is one in which there is one small parameter $\lambda / L$ and one where the solution for $\lambda / L=0$, that is, complete equilibrium of the heterogeneous system, is well defined. Therefore, it is reasonable to seek a perturbation solution to the general nonequilibrium problem of the form

$$
\begin{aligned}
u & =u^{(0)}+\frac{\lambda}{L} u^{(1)}+\left(\frac{\lambda}{L}\right)^{2} u^{(2)}+\cdots, \\
p & =p^{(0)}+\frac{\lambda}{L} p^{(1)}+\left(\frac{\lambda}{L}\right)^{2} p^{(2)}+\cdots, \\
\rho & =\rho^{(0)}+\frac{\lambda}{L} \rho^{(1)}+\left(\frac{\lambda}{L}\right)^{2} \rho^{(2)}+\cdots, \\
T & =T^{(0)}+\frac{\lambda}{L} T^{(1)}+\left(\frac{\lambda}{L}\right)^{2} T^{(2)}+\cdots, \\
u_{\mathrm{s}}\left(\sigma_{1}\right) & =\frac{\lambda}{L} u_{\mathrm{s}}^{(1)}\left(\sigma_{1}\right)+\left(\frac{\lambda}{L}\right)^{2} u_{\mathrm{s}}^{(2)}\left(\sigma_{1}\right)+\cdots, \\
u_{\mathrm{s}}^{\prime}\left(\sigma_{1}\right) & =\frac{\lambda}{L} u_{\mathrm{s}}^{(1)}\left(\sigma_{1}\right)+\left(\frac{\lambda}{L}\right)^{2} u_{\mathrm{s}}^{(2)}\left(\sigma_{1}\right)+\cdots, \\
\rho_{\mathrm{s}}\left(\sigma_{1}\right) & =\frac{\lambda}{L} \rho_{\mathrm{s}}^{(1)}\left(\sigma_{1}\right)+\left(\frac{\lambda}{L}\right)^{2} \rho_{\mathrm{s}}^{(2)}\left(\sigma_{1}\right)+\cdots, \\
T_{\mathrm{s}}\left(\sigma_{1}\right) & =\frac{\lambda}{L} T_{\mathrm{s}}^{(1)}\left(\sigma_{1}\right)+\left(\frac{\lambda}{L}\right)^{2} T_{\mathrm{s}}^{(2)}\left(\sigma_{1}\right)+\cdots, \\
u_{\mathrm{s}}\left(\sigma_{2}\right) & =\frac{\lambda}{L} u_{\mathrm{s}}^{(1)}\left(\sigma_{2}\right)+\left(\frac{\lambda}{L}\right)^{2} u_{\mathrm{s}}^{(2)}\left(\sigma_{2}\right)+\cdots, \\
u_{\mathrm{s}}^{\prime}\left(\sigma_{2}\right) & =\frac{\lambda}{L} u_{\mathrm{s}}^{(1)}\left(\sigma_{2}\right)+\left(\frac{\lambda}{L}\right)^{2} u_{\mathrm{s}}^{\prime(2)}\left(\sigma_{2}\right)+\cdots, \\
\rho_{\mathrm{s}}\left(\sigma_{2}\right) & =\frac{\lambda}{L} \rho_{\mathrm{s}}^{(1)}\left(\sigma_{2}\right)+\left(\frac{\lambda}{L}\right)^{2} \rho_{\mathrm{s}}^{(2)}\left(\sigma_{2}\right)+\cdots, \\
& =\frac{\lambda}{L} T_{\mathrm{s}}^{(1)}\left(\sigma_{2}\right)+\left(\frac{\lambda}{L}\right)^{2} T_{\mathrm{s}}^{(2)}\left(\sigma_{2}\right)+\cdots,
\end{aligned}
$$

where the basic gas-dynamic variables have zeroth order parts while the slip quantities, those giving the difference between actual particle states and those particle states that would obtain under conditions of equilibrium, are of first and higher order in $\lambda / L$, the expansion parameter.

Substitution of the assumed expansion solution into the twelve appropriate equations yields equations that may be separated into groups of various orders, according to the power of $\lambda / L$ that appears as a factor. The zeroth order solution is given by the zeroth order parts of Eqs. (42), (44), (45), and (49) alone; these are, respectively,

$$
\begin{gathered}
\rho^{(0)} u^{(0)} A=\dot{m}, \\
\bar{c}_{p} T^{(0)}+\frac{1}{2} u^{(0)^{2}}=\mathrm{const}, \\
p^{(0)}=\rho^{(0)} R T^{(0)}, \\
T^{(0)} / T_{0}=\left(p^{(0)} / p_{0}\right)^{(\bar{\gamma}-1) / \bar{\gamma}},
\end{gathered}
$$

and the result confirms the earlier conjecture that the equilibrium solution, i.e., with $\lambda / L=0$, represents the isentropic flow of a gas with modified properties.

The first order solution for the particle state, that is, the amount by which the particle state departs from the corresponding equilibrium state, follows from the lowest order parts of the remaining eight equations of the system. Equations (51) and (52) have only first order parts

$$
\begin{aligned}
& u^{(0)} \rho_{\mathrm{s}}^{(1)}\left(\sigma_{1}\right)+\kappa\left(\sigma_{1}\right) \rho^{(0)} u_{\mathrm{s}}^{(1)}\left(\sigma_{1}\right)=0, \\
& u^{(0)} \rho_{\mathrm{s}}^{(1)}\left(\sigma_{2}\right)+\kappa\left(\sigma_{2}\right) \rho^{(0)} u_{\mathrm{s}}^{(1)}\left(\sigma_{2}\right)=0,
\end{aligned}
$$

while Eqs. (53) through (58) have relevant zeroth order parts.

$$
\begin{gathered}
u^{(0)} \frac{d u^{(0)}}{d x}-\frac{\lambda}{\bar{\lambda}_{v}\left(\sigma_{1}\right)} \frac{\bar{a}^{(0)}}{L} u_{\mathrm{s}}^{(1)}\left(\sigma_{1}\right)-\kappa\left(\sigma_{2}\right)\left(\frac{\rho^{(0)}}{\rho_{0}}\right) \frac{\lambda^{2}}{l L^{2}} \\
\cdot\left|u_{\mathrm{s}}^{(1)}\left(\sigma_{1}\right)-u_{\mathrm{s}}^{(1)}\left(\sigma_{2}\right)\right|\left[u_{\mathrm{s}}^{(1)}\left(\sigma_{1}\right)-u_{\mathrm{s}}^{(1)}\left(\sigma_{2}\right)\right]=0, \\
u^{(0)} \frac{d u^{(0)}}{d x}-\frac{\lambda}{\bar{\lambda}_{v}\left(\sigma_{2}\right)} \frac{\bar{a}^{(0)}}{L} u_{\mathrm{s}}^{(1)}\left(\sigma_{2}\right)+\kappa\left(\sigma_{1}\right)\left(\frac{\rho^{(0)}}{\rho_{0}}\right) \frac{\lambda^{2}}{l L^{2}} \\
\cdot\left|u_{\mathrm{s}}^{(1)}\left(\sigma_{1}\right)-u_{\mathrm{s}}^{(1)}\left(\sigma_{2}\right)\right|\left[u_{\mathrm{s}}^{(1)}\left(\sigma_{1}\right)-u_{\mathrm{s}}^{(1)}\left(\sigma_{2}\right)\right]=0, \\
u^{(0)} \frac{d u^{(0)}}{d x}-\frac{\lambda}{\bar{\lambda}_{v}\left(\sigma_{1}\right)} \frac{\bar{a}^{(0)}}{L} u_{\mathrm{s}}^{(1)}\left(\sigma_{1}\right)=0, \\
u^{(0)} \frac{d u^{(0)}}{d x}-\frac{\lambda}{\bar{\lambda}_{v}\left(\sigma_{2}\right)} \frac{\bar{a}^{(0)}}{L} u_{\mathrm{s}}^{(1)}\left(\sigma_{2}\right)=0, \\
u^{(0)} \frac{d T^{(0)}}{d x}-\frac{\lambda}{\bar{\lambda}_{\mathrm{T}}\left(\sigma_{1}\right)} \frac{c_{p}}{c} \frac{\bar{a}^{(0)}}{L} T_{\mathrm{s}}^{(1)}\left(\sigma_{1}\right)=0, \\
u^{(0)} \frac{d T^{(0)}}{d x} \frac{\lambda}{\bar{\lambda}_{\mathrm{T}}(\sigma} \frac{\left.c_{p}\right)}{c} \frac{\bar{a}^{(0)}}{L} T_{\mathrm{s}}^{(1)}\left(\sigma_{2}\right)=0 .
\end{gathered}
$$

Notice in particular that these are six algebraic equations for the first-order slip quantities, and from 
a mathematical viewpoint the degree of the system as a set of differential equations has been depressed by six. Physically speaking, this means that one is unable to prescribe initial conditions on any of the first-order slip quantities. This result is in keeping with the earlier discussion that the transition, from arbitrary slip to one where the slip is determined by the rate of change of local gas conditions, is short in comparison with the characteristic time of the problem.

The first-order slip temperatures and collisionless slip velocities follow directly from Eqs. (72) through (75), and denoting a dimensionless length variable

$$
\xi=x / L
$$

and recalling that

$$
\begin{gathered}
\lambda / \bar{\lambda}_{v}\left(\sigma_{i}\right)=\left(\sigma / \sigma_{i}\right)^{2}, \\
\lambda / \bar{\lambda}_{\mathrm{T}}\left(\sigma_{i}\right)=(2 / 3 \operatorname{Pr})\left(\sigma / \sigma_{i}\right)^{2}
\end{gathered}
$$

these quantities follow as

$$
u_{\mathrm{a}}^{\prime(1)}\left(\sigma_{i}\right)=\left(\frac{\sigma_{i}}{\sigma}\right)^{2} \bar{M} \frac{d u^{(0)}}{d \xi}
$$

$$
T_{\mathrm{s}}^{(1)}\left(\sigma_{i}\right)=\frac{3}{2} \operatorname{Pr} \frac{c}{c_{p}}\left(\frac{\sigma_{i}}{\sigma}\right)^{2} \bar{M} \frac{d T^{(0)}}{d \xi} .
$$

These four quantities are given, therefore, in terms of the zeroth order or equilibrium solution. With this information, it is possible to solve Eqs. (70) and (71) for the average slip velocities, in contrast with the collisionless slip velocities that have just been obtained. In doing this, it is convenient to interchange the absolute value as it appears in these equations as

$$
\begin{aligned}
& \left|u_{\mathrm{s}}^{(1)}\left(\sigma_{1}\right)-u_{\mathrm{s}}^{(1)}\left(\sigma_{2}\right)\right|\left[u_{\mathrm{s}}^{(1)}\left(\sigma_{1}\right)-u_{\mathrm{s}}^{(1)}\left(\sigma_{2}\right)\right] \\
& =\left[u_{\mathrm{s}}^{(1)}\left(\sigma_{1}\right)-u_{\mathrm{s}}^{(1)}\left(\sigma_{2}\right)\right]\left|u_{\mathrm{s}}^{\prime(1)}\left(\sigma_{1}\right)-u_{\mathrm{s}}^{(1)}\left(\sigma_{2}\right)\right|
\end{aligned}
$$

as was discussed earlier. This latter term may, in fact, be written as

$$
\begin{aligned}
& {\left[u_{\mathrm{s}}^{(1)}\left(\sigma_{1}\right)-u_{\mathrm{s}}^{(1)}\left(\sigma_{2}\right)\right]\left|u_{\mathrm{g}}^{(1)}\left(\sigma_{1}\right)-u_{\mathrm{s}}^{(1)}\left(\sigma_{2}\right)\right|} \\
& =\left|\bar{M} \frac{d u^{(0)}}{d \xi}\right|\left|\left(\frac{\sigma_{1}}{\sigma}\right)^{2}-\left(\frac{\sigma_{2}}{\sigma}\right)^{2}\right|\left[u_{\mathrm{s}}^{(1)}\left(\sigma_{1}\right)-u_{\mathrm{s}}^{(1)}\left(\sigma_{2}\right)\right] .
\end{aligned}
$$

Then it follows directly from Eqs. (70) and (71) that the difference between the first-order slip velocities of the two particle clouds is

$$
u_{\mathrm{s}}^{(1)}\left(\sigma_{1}\right)-u_{\mathrm{s}}^{(1)}\left(\sigma_{2}\right)=\frac{\bar{a}^{(0)}\left[\left(\frac{\sigma_{1}}{\sigma}\right)^{2}-\left(\frac{\sigma_{2}}{\sigma}\right)^{2}\right] \Omega}{1+\frac{\lambda^{2}}{l L} \frac{\rho^{(0)}}{\rho_{0}}\left[\kappa\left(\sigma_{2}\right)\left(\frac{\sigma_{1}}{\sigma}\right)^{2}+\kappa\left(\sigma_{1}\right)\left(\frac{\sigma_{2}}{\sigma}\right)^{2}\right]|\Omega|\left|\left(\frac{\sigma_{1}}{\sigma}\right)^{2}-\left(\frac{\sigma_{2}}{\sigma}\right)^{2}\right|}
$$

where the parameter $\Omega$ has been employed to denote the dimensionless convective acceleration in the equilibrium flow.

$$
\Omega \equiv\left(u^{(0)} / \bar{a}^{(0)^{2}}\right) d u^{(0)} / d \xi
$$

Some physical feeling for the effect of collisions upon the slip velocities may be obtained directly from Eq. (83). If the collisions are very infrequent, so that the collision time $\tau_{\mathrm{c}}$ is very long in comparison with the velocity equilibration time $\tau_{v}$, then the model employed is certainly valid and $\lambda^{2} / l L \approx 0$. The result obtained is precisely the result obtained if the analysis had been carried out neglecting collisions. It demonstrates that the sign of the term $u_{\mathrm{s}}^{(1)}\left(\sigma_{1}\right)-u_{\mathrm{s}}^{(1)}\left(\sigma_{2}\right)$ is influenced by two factors: the relative size of the two particle classes, and the sign of the convective acceleration. If the collisions are sufficiently frequent that $\tau_{\mathrm{c}}$ and $\tau_{\vartheta}$ are generally of the same order, then $\lambda^{2} / l L \approx 1$ and the denominator in Eq. (83) is increased in proportion to the convective acceleration, the weighted concentrations of solid particles, and the difference in squares of the particle radii. Because of the absolute values in the denominator, the effect of collisions is always to reduce the difference between the slip velocities of the two particle classes. This is quite apparent from an intuitive standpoint. If $\lambda^{2} / l L \gg 1$, then it is implied that the collision time is much shorter than the velocity equilibration time, and the collision model is clearly invalid. It is interesting to note, however, that in a gross sense the result is correct, for in the limit as $\lambda^{2} / l L \gg 1$ the slip velocities of the two particle classes become equal. The individual slip velocities may now be calculated directly by utilizing Eq. (83) in conjunction with either Eq. (70) or (71).

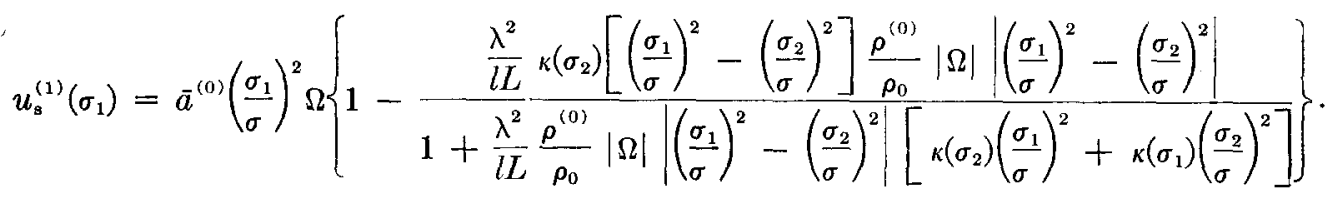


The corresponding equations for $u_{\mathrm{s}}^{(1)}\left(\sigma_{2}\right)$ may be obtained simply by changing subscripts on particle radii wherever they occur.

Again, the results of this calculation agree with the general intuitive feeling one has about the problem. For circumstances where the collision time is long in comparison with the velocity equilibration time, that is, $\lambda^{2} / l L \ll 1$, the slip velocity $u_{\mathrm{s}}^{(1)}\left(\sigma_{1}\right)$ is essentially $\bar{a}^{0}\left(\sigma_{1} / \sigma\right)^{2} \Omega$, the value which would have been obtained in the absence of collisions. When $\tau_{\mathrm{b}}$ and $\tau_{\mathrm{b}}$ are of the same order, this slip velocity is decreased if $\sigma_{1}$ is the larger particle and increased if $\sigma_{1}$ is the smaller particle. Physically, this is clear, for in drawing the two slip velocities closer together, the collision process has the effect of reducing the larger slip and increasing the smaller. The sense of this change is reversed, of course, if the local convective acceleration is negative rather than positive. Again, it is of some interest to examine the limit $\lambda^{2} / l L \gg 1$ for which the detailed mechanism employed is definitely invalid. Then Eq. (85) reduces to

$$
\begin{aligned}
u_{\mathrm{s}}^{(1)}\left(\sigma_{1}\right) & =u_{\mathrm{s}}^{(1)}\left(\sigma_{2}\right) \\
& =\bar{a}^{(0)} \Omega\left(\frac{\kappa\left(\sigma_{1}\right)+\kappa\left(\sigma_{2}\right)}{\left(\sigma / \sigma_{2}\right)^{2} \kappa\left(\sigma_{2}\right)+\left(\sigma / \sigma_{1}\right)^{2} \kappa\left(\sigma_{1}\right)}\right)
\end{aligned}
$$

which is the value when the two particle classes are constrained to move together. With these two slip velocities known, the particle density perturbations follow directly from Eqs. (68) and (69) respectively. The solution for all first-order perturbation quantities pertaining to each particle class is therefore complete.

It remains only to find the first-order perturbation solutions $u^{(1)}, p^{(1)}, \rho^{(1)}$, and $T^{(1)}$ for the gaseous component. The equations determining these quantities are the first order parts of Eqs. (42), (44), (45), and (49). These are, respectively,

$$
\begin{gathered}
\rho^{(1)} / \rho^{(0)}+u^{(1)} / u^{(0)}=0, \\
\frac{T^{(1)}}{T^{(0)}}+(\bar{\gamma}-1) \bar{M}^{2} \frac{u^{(1)}}{u^{(0)}} \\
=\alpha\left(\sigma_{1}\right)\left\{\frac{c}{\bar{c}_{p}} \frac{T_{\mathrm{s}}^{(1)}\left(\sigma_{1}\right)}{T^{(0)}}+(\bar{\gamma}-1) \bar{M}^{2} \frac{u_{\mathrm{s}}^{(1)}\left(\sigma_{1}\right)}{u^{(0)}}\right\} \\
+\alpha\left(\sigma_{2}\right)\left\{\frac{c}{\bar{c}_{p}} \frac{T_{\mathrm{s}}^{(1)}\left(\sigma_{2}\right)}{T^{(0)}}+(\bar{\gamma}-1) \bar{M}^{2} \frac{u_{\mathrm{s}}^{(1)}\left(\sigma_{2}\right)}{u^{(0)}}\right\} \\
\equiv F(\xi),
\end{gathered}
$$

$$
\begin{aligned}
& \frac{p^{(1)}}{p^{(0)}}=\frac{\bar{\gamma}}{\bar{\gamma}-1} \frac{1}{\bar{M}^{2}-1} \\
& \cdot\left\{F(\xi)-\left[1+(\bar{\gamma}-1) \bar{M}^{2}\right] G(\xi)\right\} .
\end{aligned}
$$

This solution for the gas-dynamic perturbation is, because of the manner in which $F(\xi)$ and $G(\xi)$ have been defined, formally the same as that given in Ref. 1 for heterogeneous flow with a single particle size. The solutions given by Eqs. (91) through (93) are appropriate except for problems that involve a transonic regime. In that case, the perturbation is singular at $\bar{M}=1$, that is, where sonic line occurs in the equilibrium flow. For this type of problem, such as a convergent-divergent nozzle, it is appropriate to enter one of the thermodynamic quantities, such as the pressure, as independent variable in preference to the geometric length. This singularity is then removed and the problem may be treated exactly as the corresponding problem was handled in references 2,3 , and 4 for single particle size.

\section{CONSISTENCY OF APPROXIMATION}

It is well to review at this point the assumptions that underlie the collision model that has been employed in light of the linearized solution that has been obtained. It is not possible to state, in general, 
for what values of physical quantities that enter the problem the assumptions may be met or, as a matter of fact, whether one may be assured that all conditions may be met with a consistent set of physical conditions. The simple relation between particle slip velocity and convective acceleration in the equilibrium flow for the first-order perturbation theory, however, permits making the condition definite.

The physical conditions and their analytical formulation are now as follows:

(1) Small slip. This is equivalent to saying that $\lambda / L$ is small and this is conveniently expressed as

$$
\frac{\lambda}{L} \equiv \frac{2}{9}\left(\frac{\rho_{s}}{\rho_{0}}\right)\left(\frac{\rho_{0} \sigma a}{\mu}\right) \frac{\sigma}{L} \ll 1
$$

where, for convenience, the reference particle radius is taken as $\sigma=\frac{1}{2}\left(\sigma_{1}+\sigma_{2}\right)$.

(2) The particle Reynolds number shall be of order unity or less. This becomes

$$
\operatorname{Re} \equiv \frac{\rho_{0} \sigma a}{\mu} \frac{\lambda}{L} \Omega \sim 1
$$

where $\Omega$ is the convective acceleration parameter for equilibrium flow, $\Omega=\left(u^{\langle 0\rangle} / \bar{a}^{2}\right) d u^{\langle 0\rangle} / d \xi$.

(3) The velocity equilibration time must be large in comparison with the time required for one particle to pass through the Stokes flow field of the other. Analytically, this may be written

$$
\frac{2}{9} \frac{\rho_{\mathrm{s}}}{\rho_{\mathrm{o}}} \operatorname{Re} \gg 1 \text {. }
$$

(4) The time between successive collisions of a given particle must be as large as or larger than the velocity equilibration time of the particle. This condition may be written, utilizing the previous results, as

$\frac{\tau_{z}\left(\sigma_{1}\right)}{\tau_{\mathrm{c}}\left(\sigma_{1}\right)} \equiv \frac{4}{3} \operatorname{Re} K\left(\sigma_{2}\right)\left|\frac{\sigma_{1}-\sigma_{2}}{\sigma_{2}}\right|\left(\frac{\sigma_{1}}{\sigma_{2}}\right)^{2} \leq O(1)$.

Aside from numerical factors and the mass fraction of solid particles, these relations involve five physical groups:

$$
\frac{\rho_{\mathrm{s}}}{\rho_{0}}, \frac{\sigma}{L}, \Omega, \frac{\rho_{0} \sigma a}{\mu}, \frac{\sigma_{1}}{\sigma_{2}},
$$

which may be controlled independently. Therefore, it is physically possible to choose a situation where the above restrictions are satisfied and, in addition, the collisional force parameter $\lambda^{2} / L L$ is of order unity. This collisional force parameter may be written explicitly as

$$
\frac{\lambda^{2}}{l L}=\frac{\operatorname{Re}}{12 \Omega} \frac{\left(1+\sigma_{1} / \sigma_{2}\right)^{3}}{1+\left(\sigma_{1} / \sigma_{2}\right)^{3}} .
$$

One distinct result of the above inequalities follows from equation (96), that is, that since the Reynolds number must be of order unity, the density ratio $\rho_{\mathrm{s}} / \rho_{0}$ must be large. It appears therefore that the condition that particle collisions are independent of local particle flow fields can not usually be satisfied for solid particles in liquids but is usually satisfied for solid particles in gases.

\section{CONCLUDING REMARKS}

The model for particle collision processes in the one-dimensional flow of gas-particle mixtures provides at least a first step into this branch of fluid mechanics. It does handle adequately the circumstances where the collisional and viscous forces on a particle cloud are of the same order or smaller and does give fairly straightforward criteria for the applicability of the model. The model is inadequate, however, to deal with solids in liquids unless the Reynolds number limit is relaxed and the drag law for the particles modified appropriately.

It seems probable that the general results of the analysis may hold into regions of shorter collision times than permit the model to be strictly applicable. For the essential ingredients of the collisional problem are (i) the momentum exchange depends quadratically upon the relative particle velocities; (ii) the momentum exchange depends upon the product of concentration of the two particles; and (iii) a length $l$ related to the average spacing of colliding particles. Although the proposed model serves to calculate the characteristic length in a well-defined manner, any other model, including one valid for shorter collisional time, would also lead to a definite characteristic length that would enter the problem in the same manner as the one now employed. It seems, therefore, that the result may be of somewhat wider applicability than the hypotheses would indicate.

\section{ACKNOWLEDGMENT}

This work was supported by the National Science Foundation Grant GP-713. 